\title{
DIE POLITISCHE VERPFLICHTUNG: GRÜNDE UM UNABHÄNGIG VOM WILLEN ZU HANDELN
}

\author{
Isabel Victoria Lucena Cid \\ Universität Pablo de Olavide, Sevilla
}

\begin{abstract}
This article analyzes Searle's thesis according to which all political power represents a deontic power related to rights, duties, obligations, authorizations, permissions, authority, etc. This conception leads to understand to us the power and the constituent functions of status irremediably tie to the collective acceptance and constitutive rules. Notions like linguistic constitution of deontic powers, the reasons to act independently of desire, the normative rationality and the collective acceptance of the political systems, constitute some of angular stones of a conceptual frame whose social, institutional and politic realistic vision have important implications in the scope of the legal, political and moral philosophy.
\end{abstract}

\begin{abstract}
Abstrac.- Dieser Artikel analysiert Searles Theorie, nach der jede politische Macht auch eine deontische Macht ist, die mit Rechten, Pflichten, Obligationen, Erlaubnissen, Autorität, usw. verbunden ist. Dieses Konzept bringt uns dazu zu verstehen, dass die Macht und die Statusfunktionen unmittelbar mit der kollektiven Akzeptanz und den konstitutiven Normen verbunden sind. Begriffe, wie zum Beispiel die linguistische Konstitution der deontischen Macht, die Gründe unabhängig vom Willen zu agieren, die normative Rationalität und die kollektive Akzeptanz der politischen Systeme, bilden eine Art konzeptueller Rahmen dessen soziale, institutionelle und politische Vision wichtige Auswirkungen auf den Anwendungsbereich der legalen, politischen und moralischen Philosophie haben.
\end{abstract}

Key words.- Policy, deontic power, institutional facts, political commitment, normative rationality, political system, collective acceptance, function of status

\section{Einleitung}

Im Jahre 2003 veröffentlichte John Searle seine Arbeit mit dem Titel "Social Ontology and Political Power" (Schmitt, 2003, 195-210) und im selben Jahr erschien unter dem Titel "Ontlogia sociale. Potere deontico e regole constitutive" (Sealer, 2003, 27-44) ein Gemeinschaftswerk von Paolo di Lucia. Searle hatte zum Ziel die "Ontologie der politischen Macht” und die „Rolle der Sprache in der Konstitution der besagten Macht" zu erklären. Das Hauptpostulat seines Buches „Die Konstruktion der gesellschaftlichen Wirklichkeit: zur Ontologie sozialer Tatsachen" impliziert eine politische Ontologie, oder zumindest eine Beziehung zwischen einer möglichen politischen Philosophie und einer Philosophie der Sprache. Die Frage, die er bei dieser Gelegenheit stellte, sollte beantworten wie wir als denkende, intelligente, freie, gesellschaftliche und politische Wesen eine bestimmte Auffassung, die wir von uns selbst haben, mit der Weltanschauung einer Welt, die aus physischen Teilchen in Kraftfeldern besteht, die keinerlei Intelligenz oder Bedeutung haben, in Einklang bringen können. In dieser neuen Arbeit geht er noch einen Schritt weiter und stellt erneut dieselbe Frage, aber jetzt mit einem neuen Element: Wie kann es in einer Welt, die aus physischen Teilchen besteht, eine politische Wirklichkeit geben? 
Um diese Frage beantworten zu können, nimmt Searle die ontologischen Postulate, die er in seinem Buch „Die Konstruktion der gesellschaftlichen Wirklichkeit: zur Ontologie sozialer Tatsachen" beschrieben hat, wieder auf und stellt von neuem die ontologischen und epistemologischen Unterschiede auf, die ihm schon als Grundlage zur Erklärung der institutionellen Tatsachen und der institutionellen Wirklichkeit dienten. $\mathrm{Er}$ legt seine Argumente folgendermaßen dar: gewisse Elemente der Wirklichkeit, wie z.B. Kraft, Masse, Anziehungskraft, Fotosynthese oder chemische Bindungen sind beobachterunabhängig, während andere Elemente beobachterabhängig sind und ihre Existenz von der Einstellung, dem Denken und der Absicht der Beobachter, Benutzer, Kreativen, Designer, Käufer, Verkäufer, oder etwas genereller ausgedrückt, der bewussten, beabsichtigten Kräfte abhängt. Beobachterabhängige Phänomene sind u.A. Geld, Eigentum, Heirat und Sprache (Searle, 2003, 28).

Searle fügt der epistemischen Sachlichkeit und Subjektivität auch noch die ontologische Sachlichkeit und Subjektivität hinzu. Sowohl die epistemische Objektivität als auch die epistemische Subjektivität sind Eigenschaften der Assertion, die in diesem Sinne auch als unabhängig von Gefühlen, Handlungen, Vorlieben, usw. derjenigen die sie machen oder auslegen, angesehen werden kann. „Zapatero ist der jetzige Ministerpräsident von Spanien” ist zum Beispiel eine epistemologisch sachliche Aussage, während die Aussage „Zapatero ist ein besserer Ministerpräsident als sein Vorgänger Aznar" epistemologisch subjektiv ist. Was nun die ontologische Sachlichkeit und Subjektivität angeht, so sind dieses Eigenschaften der Wirklichkeit. Auf diese Weise sind z.B. Schmerzen oder Hunger ontologisch subjektiv, weil ihre Existenz von einem menschlichen oder tierischen Wesen gefühlt wird. Berge, Strände und Moleküle wiederum sind ontologisch objektiv weil ihre Existenz nicht von subjektiven Erfahrungen abhängt.

Die Rechtfertigung dieser Unterschiede im Rahmen der aktuellen Diskussion führt Searle dazu zu folgern, dass praktisch unsere ganze politische Wirklichkeit vom Beobachter abhängt. Die Wahlen, ein Parlament, ein Regierungschef oder eine Revolution, zum Beispiel, sind was sie sind nur weil die Leute eine gewisse Einstellung zum Thema haben. Aus diesem Grunde haben alle sozialen und politischen Phänomene einen Teil ontologischer Subjektivität. Das heisst aber nicht, dass die ontologische Subjektivität als solche eine epistemische Subjektivität beinhaltet. Es gibt bestimmte Bereiche, wie z.B. die Politik oder die Wirtschaft, in denen die Entitäten subjektiv ontologisch sind, obwohl man von innen epistemisch objektive Assertionen machen kann. Auf diese Weise ist dann die amerikanische Präsidentschaft ein beobachterabhängiges Phänomen und ontologisch objektiv. Im Gegenteil: die Tatsache, dass Barack Obama der jetzige US-Präsident ist, ist ein epistemisch objektiver Tatbestand (Searle, 2004, 94).

Wir beziehen uns auf die vorherigen Überlegungen und nehmen uns vor, in dieser Arbeit die Searlsche Sicht der politischen Realität zu erforschen. Aus diesem Grunde, nehmen wir wieder eine Reihe von Voraussetzungen auf, auf die Searle das Kernstück seiner politischen Ontologie stützt: jede politische Macht ist eine Frage der Statusfunktionen und aus diesem Grund ist jede 
politische Herrschaft, eine deontische Macht. Da jede politische Herrschaft eine Frage der Statusfunktion ist, kommt sie, selbst wenn sie von oben ausgeführt wird, von unten. Die Statusfunktionssysteme funktionieren, zumindest teilweise, weil wir die deontischen Kräfte anerkennen und aus diesem Grund unabhängig von unseren Wünschen handeln. Diese Prinzipien bieten uns, wie wir noch sehen werden, eine Erklärung oder einen Beweis für den konzeptuellen Rahmen in dem man den Schritt von rohen in soziale und institutionelle Tatsachen nachvollziehen kann und von dort in das Spezifikum der politischen Tatsachen.

\section{2.- Searles Auffassung der politischen Macht}

Searles Vorhaben in seiner Arbeit "Social Ontology and Political Power” ist es nicht, etwas zu der Debatte über die westliche politische Philosophie beizutragen, sondern einige bestehende Beziehungen zwischen der Ontologie der sozialen Realität und der spezifischen Form der sozialen Realität, die die politische Herrschaft voraussetzt, zu erforschen. Einige der Grundkenntnisse die er benutzt, um die Natur der gesellschaftlichen und institutionellen Wirklichkeit zu beschreiben und zu analysieren, könnten uns helfen, uns ein Bild von der Natur der politischen Wirklichkeit zu bilden. Sein Ziel ist es, zu zeigen, dass die politische Herrschaft ein spezieller Fall der sozialen und institutionellen Wirklichkeit ist. Aus diesem Grund heben wir vier Kategorien, die uns bei diesem Vorhaben helfen können, hervor. Die Erste ist die Statusfunktion, zusammen mit den institutionellen Fakten und deontischen Mächten. Der Begriff der kollektiven Akzeptanz, die die Statusfunktionen bildet und erhält, ist in der zweiten Kategorie mit diesen Vorstellungen verbunden. Die dritte Idee ist mit der Rolle der Sprache im Aufbau der gesellschaftlichen und politischen Phänomene verknüpft. Die vierte Kategorie hat etwas mit der arteigenen Fähigkeit der Menschen zu tun, Gründe zu suchen um unabhängig vom eigenen Wunsch zu handeln. Laut Searle haben Menschen die Gabe, Handlungsgründe zu erkennen und sich selbst zu motivieren, selbst wenn diese Handlungsgründe unabhängig von den „primären Interessen” sind. Diese Kapazität ist einer der Hauptgründe für die Existenz der menschlichen Zivilisation und ausserdem ist sie einer der Gründe, der es uns ermöglicht uns politisch zu organisieren (Searle, 2003, 30). Obwohl jede einzelne dieser Ideen sich selbst erklärt, brauchen wir sie doch alle, um Searles ontologische Vorstellung der politischen Herrschaft zu verstehen.

Wir werden die Postulate anhand der genannten Argumente untersuchen, damit wir Searles Frage beantworten können, die er wie folgt stellt: Was muss den gesellschaftlichen Tatsachen hinzugefügt werden, damit sie zu politischen Handlungen werden?

\subsection{Die Politische Herrschaft als Deontische Macht}

Jede politische Herrschaft stellt eine Frage des Status dar und aus diesem Grund ist jede politische Herrschaft eine deontische Macht. Dieses Postulat repräsentiert den Grundstein, auf den sich die anderen Prinzipien, die Searles 
Buch „Die Konstruktion der gesellschaftlichen Wirklichkeit: zur Ontologie sozialer Tatsachen" ausmachen, stützen. Die deontischen Mächte haben, unter anderem, etwas mit Rechten, Pflichten, Verantwortlichkeiten, Autorisierungen, Erlaubnissen, Autorität zu tun. Die Macht der Leiter der lokalen politischen Partei und des Gemeinderates, ebenso wie die Macht der großen Figuren (Präsidenten, Regierungschefs, Kongressmitglieder oder Richter am Obersten Gerichtshof) sind eine Folge daraus, dass diese Körperschaften anerkannte Statusfunktionen innehaben (Searle, 2004, 108).

In seinem Buch „Die Konstruktion der sozialen Wirklichkeit” benutzt Searle die Terminologie der herkömmlichen Macht, um sie von der rohen Macht zu unterscheiden, obwohl, wie er selbst zugibt, eine herkömmliche Macht zuzugestehen manchmal heißt, die rohe Macht zu autorisieren, so wie es in Ausnahmenfällen bei den Staatssicherheitskräften vorkommt. Trotzdem argumentiert Searle, dass die Nutzung von Gewalt vonseiten der Polizei und der militärischen Kräfte sich der politischen Herrschaft gegenüberstellt. Ebenso können Konflikte zwischen ethnischen und religiösen Gruppen, Gewalt, die durch verschiedene Ideologien ausgelöst wird sowie der internationale Terrorismus und nicht erklärte Kriege zwischen Staaten, nicht als politisch betrachtet werden. Auf diese Weise stellt Searle die logische Struktur der deontischen Macht der logischen Struktur der Macht, die auf Kraft beruht, gegenüber.

Oppenheim ist nicht einverstanden mit diesem Argument und zeigt seine Unstimmigkeit gegenüber der Tatsache, dass die rohe körperliche Macht des Starken gegenüber dem Schwachen anders ein soll, als die politische Herrschaft (Oppenheim, 2003, 105). Er geht noch einen Schritt weiter und betrachtet die Charakterisierung, die Searle von der politischen Herrschaft als wesentlich deontisch, nicht gewalttätig und nicht egoistisch nennt, als eine idealisierte Darstellung der westlichen Demokratien.

Es stimmt, dass in den demokratisch konstitutionellen Systemen die Individuen der Ursprung jeder politischen Macht sind, aber das ist nur in der Theorie so. Laut Oppenheim beruhen fast alle (wenn nicht sogar alle) Ausübungen der politischen Macht, und die Reaktion auf dieselben, auf persönlichen Interessen. Außerdem werden Konflikte politischer Natur normalerweise mittels Anwendung roher Macht (Oppenheim, 2003, 106) oder überhaupt nicht gelöst.

Die Gegenüberstellung von deontischer und roher Macht beruht auf dem Vergleich von institutionellen (Alarcón, 2001, 33) und rohen Tatsachen, gemäß dieses Vergleiches nimmt die logische Basisstruktur der institutionellen Tatsachen die Form „X gilt im Kontext C als $Y$ ” an. Diese Formel hebt die konstitutiven Regeln, die uns ermöglichen institutionelle Tatsachen zu erschaffen, hervor. Searle bietet noch eine andere Formel an, die die Schaffung der Grundmächte in der Gesellschaft erklärt. Er konzentriert sich dabei auf die allgemeine Form des Inhaltes der Statusfunktion von $Y$. Wenn der Inhalt von $Y$ dem Element $X$ durch allgemeine Akzeptanz auferlegt wird, müssen diese allgemeinen Akzeptanzen (Anerkennung, Glauben, usw.) einen Inhalt haben. Searle sagt, dass für eine große Anzahl von Fällen dieser Inhalt mit irgendeiner Art von konventioneller Macht zu tun hat, in der das Subjekt mit einer Tat oder 
einem Tatablauf in Verbindung gebracht wird. Da die Macht immer den Einfluss hat etwas zu tun oder zu verhindern dass jemand anderes etwas tut, ist der Inhalt der Statusfunktionen der Macht immer teilweise (S tut A) (Searle, 1997, 116), wobei $S$ eine Person repräsentiert und $A$ eine Tat oder eine Aktivität. Die ursprüngliche Struktur der kollektiven Intentionalität $X$ gilt als $Y$ in $C$, wird jetzt zur Grundstruktur der konventionellen Macht: Wir akzeptieren $S$ hat die Macht (S tut $A$ ).

Welche Beziehung besteht zwischen diesen beiden Formeln: $X$ gilt als $Y$ in $C$ und wir akzeptieren, dass $S$ die Macht innehat ( $S$ macht $A)$ ? Searle gibt zur Antwort, dass wir nicht nur akzeptieren, dass jemand die Macht innehat, sondern dass wir außerdem billigen, dass diese Person die Macht aufgrund eines institutionellen Status besitzt. Ein Beispiel von $X$ gilt als $Y$ im Kontext $C$ wäre folgendes: man muss verschiedene Bedingungen erfüllen, um zum Präsidenten eines Staates zu werden. Aber wenn wir erst akzeptiert haben, dass jemand der Präsident ist, akzeptieren wir auch, dass er die Macht besitzt, um gewisse Dinge zu verwirklichen. Er hat die positive Macht, über die Streitmächte zu herrschen, er hat aber auch die negativen Mächte, das heißt, die Pflicht die Nation zu regieren. Er hat das Recht über das Militär und die Sicherheitskräfte zu bestimmen und die Pflicht die Regierung zu führen. Die Emphase über die kollektive Akzeptanz spiegelt sich in der Analyse wieder, die Searle über die konventionelle Macht erstellt: wir billigen, dass $S$ die Macht besitzt ( $S$ macht $A$ ) weil $S=X$, wir stimmen überein, dass $X$ als $Y$ gilt und dass die Statusfunktion die Anerkennung der deontischen Mächte beinhaltet (Searle, 2006, 18).

Searle unterscheidet zwischen verschiedenen Arten von konventionellen Mächten: symbolisch, deontisch, ehrenamtlich und verfahrensmäßig. In unserem Fall konzentrieren wir uns auf die konventionell normative, also die deontische Macht. Das heißt, wir beschränken uns auf die Mächte, denen eine spezielle Statusfunktion mit Auswirkungen im Bereich der Rechte, der Verantwortungen, Verpflichtungen, Pflichten, Privilegien, Bevollmächtigungen, der Bestrafungen, Genehmigungen, Erlaubnisse und anderen deontischen Phänomenen zugeteilt wurde. Searle geht davon aus, dass der Status Y Macht erteilt (oder verweigert) und ordnet diese deontischen Phänomene in zwei Kategorien: die positiven und die negativen Mächte. Die erste Kategorie ist der Fall, wenn das Agens mit irgendeiner Macht, einem Zertifikat, einer Genehmigung, einer Bevollmächtigung, einem Recht, einer Erlaubnis oder einer neuen Qualifikation ausgestattet wird, die es befähigen, etwas, was es andernfalls nicht machen könnte, zu tun. Es geht um die negativen Mächte, wenn das Agens bestraft wird, an eine Pflicht gebunden ist, ihm etwas vorgeschrieben wird, man ihn ersucht etwas zu tun, oder wenn es in irgendeiner Weise gezwungen wird etwas zu tun, was sonst nicht möglich wäre (Searle, 1997, 116; Alarcón, 2001, 104).

Wenn wir nicht die sozialen Objekte, wie etwa Geld, Universitäten oder Staaten Betracht ziehen, sondern die Agens, die auf diese Objekte einwirken, ergibt sich ein Unterschied zwischen dem was das Agens machen kann und was es machen soll (oder nicht machen soll), und dem was es zu tun fähig ist und dem was von ihm, als Ergebnis der Statuszuweisung die durch $Y$ bestimmt ist, zu tun 
verlangt wird (Searle, 1997, 113). Searle fügt diesem Unterschied zwischen positiven und negativen Mächten noch zwei weitere Differenzen hinzu, und zwar zwischen der Kreation und der Zerstörung von herkömmlichen Mächten und den herkömmlichen, verfahrensmäßigen und endgültigen Mächten. Ein Beispiel dafür wäre der Unterschied zwischen einer bestimmten Anzahl von Stimmen erhalten und einer Wahl gewinnen. Stimmen zu erhalten ist ein Schritt auf dem Weg zum Sieg, und gewinnen ist das Ende des Wahlvorgangs. Wir können also beobachten, dass die verfahrensmäßigen Statusfunktionen deontisch bedingte Funktionen sind, d.h. wenn die Bedingung erfüllt wird, ist das Ergebnis ein Schritt in der Hierarchie der wiederholten institutionellen Realität.

Was nun die Kreation und die Zerstörung von Mächten angeht, diese Taten können kraft anderer herkömmlicher Mächte realisiert werden, wie es z. B. der Fall bei einer Eheschließung/ Scheidung wäre. Das heißt, dass in dem Fall in dem eine Handlung die Kreation oder die Zerstörung der Macht impliziert, diese Tat die Ausführung der herkömmlichen Macht ist. Bei der Zerstörung einer herkömmlichen Macht geht die Negation über die kollektive Akzeptanz und nicht über den Inhalt der Akzeptanz (Searle, 1997, 118).

Als Folgerung seiner Forschung über die Klassifikation der institutionellen Mächte in symbolische, deontische, ehrenamtliche und verfahrensmäßige Mächte, schließt er, dass aus der Sicht einer logischen Struktur der sozialen Wirklichkeit, wir nur die Kreation und die Zerstörung von herkömmlichen Mächten haben, die symbolisch, ehrenamtlich, negativ, positiv oder bedingt sein können. Manche von diesen Mächten sind kollektiv und wieder andere individuell und repräsentieren Variationen von Iterationen der Basisstruktur (Searle, 1997, 122).

Aufgrund dieser Argumente erwägt Searle, dass die Basisstruktur der Macht uns erlaubt, alle logischen Merkmale des intentionellen Inhaltes des Funktionsstatus $Y$ in der Formel „X gilt als $Y$ ” zu erkennen und zeigt uns somit dass die enorme Komplexität des Körpers der institutionellen Realität ein ganz einfaches Skelett hat (Searle, 1997, 123). Dahingegen, und trotz der logischen Kohärenz seiner Erklärung, kommt eine Problematik über die Unterscheidung zwischen positiven und negativen Mächten, sowie über die Analogie, die Searle zwischen der deontischen Dimension und der Machtdimension und zugleich Autorität und Macht präsentiert, auf. Auf der anderen Seite ist auch die Rolle, die die konstitutiven Regeln in der logischen Struktur der herkömmlichen Macht spielen, nicht klar definiert. Laut Searle übertragen die konstitutiven Regeln die Macht durch die Zuschreibung eines Status und einer Funktion. Das Problem ist aber, dass Searle nicht erklärt wie genau das vor sich geht. Es ist zwar wahr, dass er theoretische Elemente, die die Mechanismen von Funktionszuweisung die sich auf die Formel 'count as' basieren, zeigt, aber er legt nicht im Einzelnen dar, wie die konstitutiven Regeln, die Macht verleihen, funktionieren. Er zeigt zum Beispiel nicht ausführlich genug, wie eine konstitutive Regel der Art "A gilt als Zelebrant einer Hochzeitszeremonie", A die (allgemein akzeptierte) Macht verleiht, zwei Menschen in Ehe zu verbinden. 
Den vorherigen Aussagen über Searles Sicht der konventionellen Macht folgend, können wir schließen, dass Searle sich der traditionellen Denklinie über die Macht als deontische Fähigkeit und Statusfunktion anschließt. Diese Statusfunktionen kann man in zwei umfassende Machtkategorien gliedern: die positiven und die negativen Mächte. Die erste Kategorie ergibt sich, wie wir schon gesehen haben, wenn das Agens eine bestimmte Macht innehat, die es befähigt, etwas, was es andernfalls nicht tun könnte, auszuführen. Die zweite Kategorie ergibt sich, wenn das Agens an eine Pflicht gebunden ist, ihm etwas vorgeschrieben wird, man es ersucht etwas zu tun, oder wenn es in irgendeiner Weise gezwungen wird etwas zu tun, was auf eine andere Art nicht möglich wäre.

\section{Gründe um unabhängig vom Willen zu handeln}

Ebenso wie schon vorher definiert, bilden wir die gesellschaftliche und politische Wirklichkeit in dem wir ihr Statusfunktionen zuteilen, kollektiv akzeptieren und vor dem Hintergrund (Searle, 1997, 140) beibehalten. Wir konnten schon vorher feststellen, dass die Funktionen keine eigenen Eigenschaften der Welt sind, es sind immer betrachterabhängige Merkmale. Die beiden Funktionsarten die Searle beschreibt sind die agentive und die inagentive Funktion.

Die erste Art der Funktionen ist die, die mit den Funktionen zu tun hat die wir den verschiedenen Objekten und Phänomenen der Wirklichkeit zuweisen. Es sind die agentiven Funktionen die Searle "Statusfunktionen" nennt und deren Aufgabe es ist, zu „repräsentieren”, „symbolisieren”, „zu verstehen zu geben” und allgemein zu "bedeuten”. Zusammen mit dieser Bedeutung entwickelt Searle das Konzept der desire-independent reasons for actions im Rahmen der Nicod Konferenzen die er im Jahre 2000 im Collége de France in Paris abgehalten hat. Diese Konferenzen erschienen später in einer ausgefeilteren Version unter dem Titel Freedom and Neurobiology: Reflections on Free Will, Language, and Political Power (Searle, 2000). Als er die besagte Konferenz gab und das Buch schrieb, erschien nichts über die Tatsache, dass bestimmte Dinge ein Grund dafür sein können, um unabhängig vom eigenen Willen zu handeln ohne dass sich das Agens dessen bewusst ist. Jetzt, im Kontext seiner Ontologie der politischen Macht, schlägt er vor, diese beiden Gedanken anzunähern und besteht darauf, dass die Statusfunktionen, die er in "Die Konstruktion der gesellschaftlichen Wirklichkeit: zur Ontologie sozialer Tatsachen" beschrieben hat, genau die Gründe sind, um unabhängig vom Willen zu handeln. Daraus folgt, dass die Anerkennung der Statusfunktionen auf die sich die politische Macht stützt, in sich selbst die Anerkennung von diversen Gründen ist, um unabhängig vom Willen zu handeln. Auffallend ist hier, dass das komplette soziale und politische System sich auf die menschliche Fähigkeit stützt, willensunabhängige Gründe zu erkennen und aufgrund von diesen zu handeln.

Wir wollen dieses Postulat Searles ein wenig vertiefen. Haben wir als Basis die rohen Tatsachen, dann repräsentieren die institutionellen und die politischen Tatsachen den Rahmen in dem unsere Aktivitäten sich sowohl individuell als 
auch kollektiv abspielen. Davon ausgehend, versucht Searle eine Rationalität zu definieren, die unsere Fähigkeit erklärt, bestimmte Dinge als Handlungsanstoß zu erkennen. Aber worauf basieren sich die Gründe willensunabhängig zu handeln? Und worauf beruht sich, dass $X$ als Grund für $Y$ gilt? (Liz, 2002, 88, Rodríguez, 2003, 283). Searle widersetzt sich dem klassischen Rationalitätsmodell der menschlichen Handlungen nach dem die rationalen Taten nur durch einen Wunsch oder bestimmte Interessen ausgelöst werden (Bejarano, 2001M 259). Entgegen dieser Sicht vertritt Searle, dass eine Kluft zwischen den Glauben und Wünschen einerseits und der Aktion andererseits besteht: die Kluft in der die rationale Entscheidungsfällung eine Rolle spielt, ist in Wirklichkeit dreifach. Die erste Kluft nehmen wir zwischen den Gründen für unsere Handlung und unserer tatsächlichen Entscheidung wahr. Die zweite Kluft befindet sich nach der Entscheidungsfällung, wenn man aber noch überlegen muss wann und wie man sie erfüllt. Zuletzt, im Fall der Aktivitäten die sich auf einen längeren Zeitraum beziehen, gibt es noch eine Kluft zwischen dem Anfang der Aktion und der kontinuierlichen Ausführung der Tat bis zur freiwilligen Vollendung (Searle, 2000, 27).

Das klassische Modell, das sich auf die Theorien der rationalen Entscheidung oder der instrumentalen Konzeption der Rationalität beruht, gilt nur für die erste Art von Gründen, d.h. für diejenigen, die wunschabhängig sind und zum Beispiel Interessen, Absichten, Vorlieben, Nutzbarkeiten, akzeptierte Werte usw. beinhalten. Dieses Modell basiert auf sechs Grundprinzipien: a) die rationalen Taten werden durch Glauben und Wünsche verursacht; b) die Rationalität beruht darauf, gewissen Regeln zu folgen; c) die Rationalität ist eine kognitive Fähigkeit die von anderen trennbar ist; d) Fälle von Willensschwäche oder akrasis, sind buchstäblich unmöglich; e) die praktische Rationalität muss immer Wünsche, Absichten, Ziele und Interessen usw. voraussetzen; f) Rationalität ist nur dann möglich, wenn diese Primärwünsche konsistent sind (Liz, 2002, 20).

In seinem Buch „Rationality in Action”, erschafft Searle neue Konzepte und zeigt verschiedene Unterschiede auf, die uns später helfen werden, sein Postulat über die Willensfreiheit zu verstehen. Einer dieser Unterschiede ist der zwischen den äußeren und den inneren Handlungsgründen. Die ersten sind faktitive Entitäten, die als Grund für ein Agens fungieren können, wenn sie angemessen als Gründe anerkannt und akzeptiert werden. Wenn dieses geschieht, wird der Grund zu einem inneren Grund. Der andere Unterschied ist mit den ganzen und partiellen Gründen verbunden. Die ganzen Gründe müssen mindestens einen Motivator haben, d.h. dass die Handlungsgründe Gruppen von faktitiven Elementen sind, die in die Anpassungsrichtung Welt-bis-Verstand arbeiten. Es können sowohl interne Motivatoren sein, wie z. B. Wünsche, Hoffnung, Furcht, Scham, Stolz, Ekel, Durst, Hunger, Freude usw., als auch externe Motivatoren. Die externen Motivatoren beinhalten Pflichten, Notwendigkeiten, Verpflichtungen, Verbindlichkeiten usw. Motivatoren sind ontologisch subjektiv weil sie von einem Subjekt abhängen, während sie aber gleichzeitig auch epistemisch objektiv sein können (Liz, 2002, 93; Searle, 2000, 140). 
Wir bilden Gründe, um wunschunabhängig zu handeln und schaffen externe Motivatoren die uns, welches auch immer unsere Wünsche sein mögen, implizieren. Im Fall der sozialen und politischen Institutionen erkennen wir Statusfunktionen als Handlungsgründe an, $z u$ denen wir uns in der gesellschaftlichen Wirklichkeit, manchmal sogar über unsere Wünsche hinaus, verpflichtet fühlen. Aus dieser Sicht, verwandeln sich die anerkannten Statusfunktionen in Gründe für eine wunschunabhängige Tat. Laut Liz Gutiérrez werden sie, sobald sie als Motivatoren anerkannt werden, auch gleich als Handlungsgründe erkannt und von diesem Moment an wägt das Agens die Kluft $\mathrm{ab}$, zieht verschiedene inm bekannte Handlungsgründe in Betracht und bildet eine Handlungsabsicht (Liz, 2002, 93; SEARLE, 2000, 142).

Die soziale Welt einerseits und die politische Welt auf der anderen Seite sind voll von faktitiven Entitäten die wie externe Motivatoren funktionieren und uns auffordern, sie als solche anzuerkennen (politische Verpflichtungen können verschiedene Gründe, um wunschabhängig zu handeln, vertreten). Die sozialen und die politischen Institutionen bilden ein vielschichtiges Flechtwerk von Entitäten mit Pflichten, Verpflichtungen, Forderungen, Verbindlichkeiten und Notwendigkeiten usw. Diese Handlungsgründe, externen Motivatoren oder Statusfunktionen die kollektiv und absichtlich geschaffen wurden, müssen erkannt, akzeptiert und beibehalten werden.

Trotzdem handeln die Menschen nicht immer aus Motiven, die unabhängig von ihren Wünschen sind. Ein Beispiel dafür können wir im Rahmen der logischen Verbindungen zwischen politischer und wirtschaftlicher Macht finden. Sowohl die wirtschaftlichen, als auch die politischen Systeme sind Statusfunktionssysteme. Wie wir gesehen haben, gehört zum politischen System die Regierungsmaschinerie. Das wirtschaftliche System aber gehört zum Wirtschaftsgetriebe welches Güter schafft und verteilt. Und obwohl die jeweiligen logischen Strukturen sich ähneln, sind die rationalen und motivationalen Systeme doch von Grund auf verschieden. Die ökonomische Macht ist essenziell mit der Fähigkeit verbunden, wirtschaftliche Vorteile und Sanktionen zu verteilen. Bei der politischen Macht ist das auch oft, aber nicht immer der Fall, vor allem in den Staaten, die starke Wohlfahrtspolitiken und Ausgleichszahlungen für benachteiligte Gruppen entwickeln. Searle ist der Meinung, dass diese Tatsache ausgelöst hat, dass eine Menge konfuser Theorien veröffentlicht wurden, die versuchen, die politischen Verhältnisse zu untersuchen, als hätten sie dieselbe logische Struktur wie die ökonomischen. Aber die Handlungsgründe die aus Wünschen oder Interessen entstehen, sind, selbst wenn sie sich auf ein deontisches System stützen, nicht deontologisch. Ein wichtiger Punkt ist es, darauf zu beharren, dass eine politische Macht essenziell eine deontische Macht ist. Diese Erkenntnis hat ihren Ursprung übrigens bei den Theoretikern des Gesellschaftsvertrages. Diese dachten, dass es kein System von politischen Pflichten und keine politische Gesellschaft ohne ein Versprechen geben könnte. Ein Versprechen oder eine Verpflichtung das nötige deontische System zu schaffen, um die politische Wirklichkeit beibehalten zu können. Was nun die politische Sphäre angeht, so sind wir nicht genötigt, ausdrücklich Gründe, um unabhängig von unseren Wünschen zu handeln zu suchen, wie etwa wenn wir etwas versprechen oder andere persönliche Verpflichtungen eingehen. Schon allein dass wir verschiedene 
institutionelle Tatsachen als gültig oder obligatorisch ansehen, schafft Gründe, die von unseren Wünschen unabhängig sind.

Auch wenn es stimmt, dass die deontischen Mächte (die Pflichten, Verpflichtungen, Forderungen, Auferlegungen usw.) Gründe für wunschunabhängige Aktionen sein könnten, so hält Oppenheim doch daran fest, dass die Beziehungen der institutionellen Mächte gleichzeitig auch persönliche Interessen implizieren. Das gilt vor allem wenn wir unsere Aufmerksamkeit auf die Macht richten, die von der politischen Gewalt (Regierungen) über die Bürger ausgeübt wird. Die Bürger führen die juristischen Normen aus dem einfachen Grund aus, weil sie die Staatsgewalt anerkennen, weil sie Strafen vermeiden wollen oder weil das Recht mit ihren persönlichen Interessen übereinstimmt (Oppenheim, 2003, 106).

\subsection{Zu einem Modell normativer Rationalität}

Kommen wir noch einmal zurück auf Searles Argument in dem er behauptet dass wir alle uns, selbst wenn wir uns dessen nicht bewusst sind, rational verhalten ohne die Normen, die die Rationalität bestimmen, zu kennen, ja sogar ohne zu wissen, dass wir bestimmten Normen folgen. Rationalität wird nicht durch eine Reihe von Normen definiert oder konstituiert (SEARLE, 2000, 35). Wenn wir hiervon ausgehen, existieren mehrere Weisen, die Wechselwirkung zwischen sozialen Normen und Rationalität zu verstehen.

Wie wir schon gesehen haben, stützt sich die institutionelle und politische Wirklichkeit auf eine Serie von konstitutiven Normen. Diese Regeln, im Gegensatz zu den regulativen Normen, besitzen die Eigenschaft bestimmte Praktiken, die sonst nicht existieren würden, zu erschaffen. Die formelle Struktur der konstitutiven Normen ist $X$ gilt als $Y$ im Kontext $C$, d.h. dass jede physische und institutionelle Tatsache eine bestimmte Statusfunktion in einem bestimmten Kontext erfüllt. Searle hat bei verschiedenen Anlässen zum Ausdruck gebracht, dass die Individuen nicht denken „dies ist die Norm, wenn wir die anwenden, haben wir eine institutionelle Tatsache”, sondern dass diese Regeln unterschwellig sind und die logische Struktur der institutionellen Tatsachen widerspiegeln.

Diese Auffassung ist genau gegensätzlich zu den sozialen Theorien, die eine Einstellung der Individuen als " normenachtend und normenfolgend" promulgieren, d.h. dass sie die konstitutiven Normen kennen und sie bewusst anwenden. Wie kann man das regelhafte Handeln des menschlichen Benehmens erklären? Searle beantwortet diese Frage auf verschieden Arten, die von seinen Denkweisen in seinen verschiedenen Arbeiten abhängen. Er versucht dieses Problem mit seinem Hintergrundkonzept zu überwinden. Der Hintergrund besteht aus einer Reihe von pre-intentionellen Fähigkeiten und Fertigkeiten, die der Regelhaftigkeit der sozialen Welt funktional äquivalent sind. Searle vertritt die Ansicht dass, wenn unser Verhalten sich den konstitutiven Normen anpasst, wir nicht im wittgensteinschen Sinn „den Regeln folgen”. Wittgenstein äußerte, dass der Gedanke, die Normen absolut unbewusst zu beachten, in sich selbst nicht kohärent sei. Absolut unwissentlich 
eine Regel zu befolgen würde uns, laut Searle, in eine Art Zombiezustand versetzen in dem wir fähig sind, etwas meisterhaft oder geschickt auszuführen und uns gleichzeitig nicht bewusst darüber sind, was wir gerade tun. Der Einfluss des Hintergrundes auf unser Verhalten ist nicht normativ und somit folgen wir keinen Normen. Ein Verhalten das auf unbewusstem Handeln basiert, kann nicht daraus bestehen die Regeln zu befolgen, sondern, wie schon Wittgenstein bemerkte, ist ein Verhalten, das mit einer Reflexhandlung zu vergleichen ist. Die Tatsache, dass unser Verhalten sich den konstitutiven Normen anpasst, rührt daher, dass die Fähigkeiten und die Voraussetzungen des Hintergrundes durch die Normen versursacht wurden, d.h. dass der Hintergrund durch konstitutive Normen logisch strukturiert wurde. Auf diese Weise lässt sich erklären, warum wir „gemäß den Normen” handeln, ohne den „Normen zu folgen”.

Keine Theorie über die Rationalität der Subjekte hat die Tragweite des searlschen Arguments. Auf welche Art können wir die rationale oder normative Orientierung in diese Analyse einführen? Hier müssen wir uns erneut an eine von Searles frühen Arbeiten halten: Die Sprechakttheorie. Dieser Theorie zufolge, kann die illokutionäre Kraft normative Verbindungen zwischen Individuen herstellen. Diese illokutionären Verknüpfungen werden auch wie die logische Struktur der konstitutiven Regeln dargestellt, außerdem teilen sie Statusfunktionen zu und schaffen deontische Mächte (Rechte, Pflichten, Verpflichtungen), die die Form der logisch äquivalenten Regel 'wir akzeptieren, dass $S$ die Macht hat, um $Y$ im Kontext $C$ durchzuführen', haben. Auf der anderen Seite, und im Gegensatz zu einem Verhalten das durch den Hintergrund bestimmt wurde, führen diese Verbindungen zu Gründen, unabhängig von den eigenen Wünschen zu handeln, d.h. zu einer nicht instrumentalen, sondern normativen Rationalität. Es ist nicht so als würden wir den Regeln irrational oder unbewusst folgen, sondern es sind die Normen, die die Handlungsgründe bewirken. Aus dem Ganzen kann man folgern, dass es eine Erklärung gibt die keine kausale Interpretation zulässt: es handelt sich um die Erklärung die sich auf die Gründe unabhängig vom Wunsch zu handeln, stützt.

Diese Gründe sind nicht kausal für unser Verhalten verantwortlich, aber die Tatsache, dass wir ihre Gültigkeit anerkennen erklärt, ob wir nach innen handeln: es sind Gründe, die nicht darauf basieren, dass wir tun wozu wir 'geneigt' sind, sondern darauf was wir glauben tun zu müssen, weil wir sie als gültig anerkennen und nicht weil wir keine andere Wahl haben.

Was Searle also zu sagen versucht ist, dass es verschiedene gültige Gründe für rationale Handlungen gibt. Diese Motive haben ihren Ursprung aber nicht in den persönlichen Wünschen oder im eigenen Interesse, sondern in den illokutionären und sozialen Beziehungen und Verbindungen, die wir aufgebaut haben. Daraus folgend sind die sozialen Regeln nicht immer schon vorher existent, sondern etwas was wir mit unseren Sprechakten erschaffen können und wenn sie erst existieren, dann funktionieren sie wie ein externer Handlungsanstoß. Searle sieht das Befolgen der Regeln wie eine bewusste Handlung die rational angestrebt wird, aber nicht aufgrund instrumenteller, 
utilitaristischer oder zweckmäßiger Motivationen, sondern angesichts der rationellen Überzeugung, dass man infolge dieser Gründe handeln muss.

\section{Reference}

(2001) ALARCÓN, Carlos, Causalidad y normatividad, Sevilla: MAD ARTOSI, Alberto "On the Notion of an Empowered Agent" en www.cirfid.unibo.it/ agsw/lea02/pp/Artosi.pdf (2004) ARTOSI, Alberto, ROTOLO, Antonino, y VIDA, Silvia "On the logical Nature of Count-as Conditionals", en CERVENINI, C. (ed.), Proceedings of LEA. Bologna: Gedit pp. 9-34.

(2001) BEJARANO, Teresa, "Acerca de las razones para actuar de Searle", en Thémata. Revista de Filosofía, ${ }^{\circ} 26$, pp. 259-273

(2003) DI LUCIA, Paolo, Ontologia sociale. Potere deontico e regole costitutive. Macerata: Quodlibert,

(2002) LIZ GUTIERREZ, Manuel, "Forma y contenido en las razones para actuar independientemente del deseo" en Revista Laguna, vol. 10,

(2006) MILLER, Seumas "Artefacts and Colletive Intentionality" en Techné, Vol. 9 (2) Winter, pp.52-67;

(2002) NOGUERA FERRER, José Antonio, "Racionalidad y normas sociales en la teoría post-analítica de la acción" en www.selene.uab.es/_cs_gr_saps/catala/autors/xxnoguera.htm

(2003) OPPENHEIM, Felix, E. "Potere Bruto e potere deontico Una risposta a Searle" en DI LUCIA, Paolo, 2003, Ontologia sociale. Potere deontico e regole costitutive. Macerata: Quodlibert,

(2003) RODRIGUEZ GONZÁLEZ, Mariano, "Esa fuerza tan especial del compromiso" en LOGOS. Anales del Seminario de Metafísica, n 36 , pp. 283288

(2003) SCHMITT, F. F. (ed.), Socializing Metaphysics: The nature of social reality, Lanham, MD: Rowlan and Littlefield.

(2003) SEARLE, John R. "Ontologia sociale e potere político" en DI LUCIA, Paolo, Ontologia sociale. Potere deontico e regole costitutive. Macerata: Quodlibert,

(2004) SEARLE, John R. Libertad y neurobiología. Reflexiones sobre el libre albedrío, el lenguaje y el poder político, Barcelona: Paidos

(1997) SEARLE, John R. La construcción de la realidad social, Barcelona:

Paidos

(2006) SEARLE, John R. "Social Ontology: Some Basic Principles" en

Anthropological Theory, Vol. 6 (1)

(2005) SEARLE, John R. "What is an Institution? en Journal of Institutional

Economics, Volume 1, Issue 01, January, pp. 1-22

(2000) SEARLE, John R., Razones para actuar. Una teoría del libre albedrío, Oviedo: Ediciones Novel

(2001) SEARLE, John, R. "Neither phenomenological description nor rational reconstruction: Replay to Dreyfus" en Revue Internationale de Philosophie, $\mathrm{n}^{\circ}$ 217. 